\title{
CONSIDERACIONES ÉTICAS EN EL TRATAMIENTO MÉDICO A TESTIGOS DE JEHOVÁ
}

\author{
Gonzalo Cardemil H.*
}

\begin{abstract}
Resumen: Actualmente, sería impensable practicar cualquier tratamiento médico sin explicar al paciente sus complicaciones y posible morbilidad, sin mencionar tratamientos alternativos o, peor aún, obligarlo o engañarlo para efectuarle un tratamiento que rechaza.

El caso de los Testigos de Jehová es digno de considerar. La experiencia nacional e internacional cuenta con respaldo científico sólido que evidencia que son practicables las cirugías complejas sin sangre, algunas de ellas impensadas ańos atrás.

El presente artículo refiere algunas normas ético-legales, tanto chilenas como internacionales, respecto de la autonomía y no discriminación de los pacientes, y expone la experiencia del Hospital Clínico de la Universidad de Chile que, desde 1998, cuenta con un exitoso programa de atención para pacientes que no desean transfundirse.
\end{abstract}

Palabras clave: autonomía, consentimiento informado, Testigos de Jehová, cirugía sin sangre

\section{ETHICAL CONSIDERATIONS IN MEDICAL TREATMENT TO JEHOVA WITNESSES}

Abstract: Currently, it would be unthinkable to treat a patient without explaining him his complications and possible morbidity, without mentioning alternative treatments or, worst, oblige or deceive him giving treatment that he rejects.

The case of Jehova Witnesses is worth considering. National and international experience show solid scientific base for the practicability of complex surgeries without the use of blood, some of them unthinkable years before.

The present article refers to some legal-ethical norms, Chilean as well as International, referring to the autonomy and non discriminating patients, and it shows the experience of the University of Chile Hospital Clinic that, since 1988, counts with a successful health care program for patients who do not wish blood transfusions.

Key words: autonomy, informed consent, Jehova Witnesses, surgery without blood

\section{CONSIDERAÇÓES ÉTICAS NO TRATAMENTO MÉDICO A TESTEMUNHAS DE JEOVÁ}

Resumo: Atualmente, seria impensável praticar qualquer tratamento médico sem explicar ao paciente suas complicaçóes e possível mortalidade, sem mencionar tratamentos alternativos, ou, pior ainda, obrigá-lo ou enganá-lo para efetuar um tratamento com o qual ele concorde. O caso dos Testemunhas de Jeová é digno de ser considerado. A experiência nacional e internacional conta com sólidas bases científicas que evidenciam práticas de cirurgias complexas sem transfusão, algumas delas impensadas anos atrás. O presente artigo se refere a algumas normas ético-legais, tanto chilenas como internacionais, que dizem respeito à autonomia e à não discriminação de pacientes, e apresenta a experiência do Hospital Clínico da Universidade do Chile que, desde 1998, desenvolve exitoso programa de atenção a pacientes que não aceitam transfusão sanguínea.

Palavras-chave: autonomia, consentimento informado, Testemunhas de Jeová, cirurgia sem transfusão sanguínea

* Unidad Cirugía de Tórax, Hospital Clínico de la Universidad de Chile, Chile Correspondencia: gcardemil@mi.cl 


\section{Introducción}

Hasta hace poco tiempo, a los pacientes respecto de su tratamiento, posibles complicaciones o efectos adversos no era práctica médica habitual. Los pacientes eran sometidos a intervenciones o tratamientos invasivos en desconocimiento de los procedimientos y sus riesgos; la autorización firmada para llevarlos a cabo era excepcional.

Actualmente, sería impensable practicar cualquier tratamiento sin explicar sus complicaciones y posible morbilidad, sin mencionar tratamientos alternativos o, peor aún, obligar o engañar a un paciente para efectuar un tratamiento que este rechaza.

El hecho de negarse a ser transfundido por motivos religiosos concita habitualmente repudio y críticas, especialmente si los hechos son tratados periodísticamente y como casos emblemáticos. ¿Qué sucede hoy entonces con los Testigos de Jehová, que son discriminados por rechazar la transfusión como tratamiento?

Si queremos analizar razonablemente el problema, es necesario contestar las siguientes interrogantes:

1. ¿Tienen derecho los Testigos de Jehová a negarse a ser transfundidos? ¿Tiene cualquier paciente el derecho a negarse a un tratamiento propuesto?

2. Los pacientes que rechazan la transfusión, ¿̇tienen per se mayor morbimortalidad?

\section{El aspecto legal}

Respecto de la primera pregunta, situada en un plano ético-legal, citaremos la Declaración Universal de los Derechos Humanos (ONU, 10 de diciembre de 1948), la Convención para la Protección de los Derechos Humanos y Libertades Fundamentales (4 de noviembre de 1950), la Carta Social Europea (18 de octubre de 1961), la Convención para la Protección de las Personas (28 de enero de 1982) y la Convención de los Derechos del Niño (20 de noviembre de 1989). Todas ellas iniciativas internacionales para garantizar el respeto a la autonomía, libertad e intimidad de las personas(1).

En el campo particular de la medicina, la Convención para la Protección de los Derechos Humanos y la Dignidad del Ser Humano con respecto a las aplicaciones de la Biología y la Medicina: Convenio relativo a los Derechos Humanos y la Biomedicina, también conocido como Convenio de Oviedo, establece: “...el ser humano debe ser respetado como persona y como perteneciente a la especie humana", debiendo garantizarse su dignidad, respetando su autonomía(2).

Basada en dicha Convención, se promulgó en España la Ley 41, que regula la autonomía del paciente y los derechos y obligaciones en materia de información y documentación clínica(3). Acerca de la autonomía, establece que esta se ejerce con la aplicación de la doctrina del consentimiento informado, definida como “...la conformidad libre, voluntaria y consciente de un paciente, manifestada en el pleno uso de sus facultades, después de recibir la información adecuada, para que tenga lugar una actuación que afecta su salud".

El artículo 2 de la citada ley señala además que "...la dignidad de la persona humana, el respeto de la autonomía de su voluntad y de su intimidad orientarán toda la actividad encaminada a obtener, utilizar, archivar, custodiar y transmitir la información y la documentación clínica”.

En Chile, el Decreto Supremo No 42, artículo 105, vigente hasta 2005, fue reemplazado por el Decreto Supremo No 140, artículo 34, que establece como obligación para el médico tratante "...informar a los pacientes, a sus representantes legales o a los familiares de ellos sobre el diagnóstico y pronóstico probable de su enfermedad, las medidas terapéuticas o médicoquirúrgicas que se aplicarán y los riesgos que estas o su omisión conllevan, para permitir su decisión informada, así como las acciones preventivas que correspondan al paciente o a su grupo familiar. En caso de negativa o rechazo a procedimientos diagnósticos o terapéuticos por parte del paciente o su representante, deberá dejarse constancia escrita en un documento oficial del servicio".

Respecto de lo anterior, el Reglamento No 570 del Ministerio de Salud de Chile precisa: "Consentimiento para tratamiento es la autorización que una persona otorga, en forma voluntaria, a la propuesta del plan de tratamiento que le indica el médico a cargo y a sus variaciones. Esta autorización deberá estar basada en el conocimiento de los fundamentos que hacen aconsejable tal tratamiento o procedimiento, los propósitos, riesgos y efectos directos y colaterales, incluyendo las posibilidades de éxito de otras alternativas terapéuticas existentes y la factibilidad de su realización".

El nuevo Proyecto de Ley No 4398-11, que regula los derechos y deberes de las personas en relación con 
acciones vinculadas con su atención de salud, en su artículo 14, No 5 expresa: "Toda persona tiene derecho a otorgar o denegar su voluntad para someterse a cualquier procedimiento o tratamiento vinculado con su atención de salud". El ejercicio de este derecho es libre, voluntario, expreso e informado". Y agrega que “...por regla general, este proceso se efectuará en forma verbal”, precisando que en determinados casos, como "intervenciones quirúrgicas, procedimientos diagnósticos o terapéuticos invasivos, y en general para la aplicación de procedimientos que conlleven un riesgo importante para la salud del afectado", la decisión debe establecerse por escrito. En tales casos, además, deben considerarse otras alternativas de tratamiento o procedimiento propuesto.

El código de ética de la International Society of Blood Transfusion estableció en 2000: "Los pacientes deberán estar informados de los riesgos y beneficios conocidos de la transfusión de sangre y/o terapias alternativas, y tendrán el derecho de aceptar o rechazar el procedimiento". Sería bueno recordar que la transfusión es un trasplante de tejido, y que, aunque en algunos países es segura, en ninguno está exenta de riesgos(4).

Tal es la base ético-legal de la que debemos partir. La negativa a someterse a cualquier tipo de examen o tratamiento está dentro de los derechos de los pacientes. Discriminar a cualquiera de ellos por tomar decisiones de salud basados en creencias religiosas o de cualquier otra índole contraviene su autonomía y libertad individual.

Normalmente, cuando un Testigo de Jehová rehúsa transfundirse, los médicos -o mejor, las directivas hospitalarias- recurren a tribunales solicitando un Recurso de Protección. Si este es acogido, puede transfundirse al paciente en contra de su voluntad.

La intención es, sin duda, proteger el derecho a la vida. Sin embargo, en la práctica, ¿es esto efectivo?

Un estudio retrospectivo de los recursos de protección tramitados en nuestro país mostró que en todos los casos se invocó que la transfusión era esencial para la continuidad de la vida. No obstante, en el $77 \%$ de los casos esto no fue efectivo, ya porque el paciente nunca fue transfundido (por huida, traslado a otro hospital y otros) o porque, aun practicándosele la transfusión, el paciente falleció. No se registró en la ficha clínica de paciente alguno que se le advirtiera sobre los riesgos de la transfusión o sobre la posibilidad de terapias alternativas(5).

Con estos datos, nos parece que esta vía judicial es ineficiente, poco práctica, de hecho inoperante y que lleva a innecesarios enfrentamientos entre médicos y pacientes.

\section{Experiencias significativas}

La experiencia nacional e internacional en la atención de Testigos de Jehová y otros pacientes que no desean transfundirse enseńa resultados favorables en numerosas patologías, como cirugía cardíaca(6-8), cirugía hepática $(9,10)$, cirugía traumatológica(11-13), ginecológica $(14,15)$, urológica $(16,17)$, trasplantes cardía$\cos (18)$, hepáticos(19-21) y renales (22,23). También, en tratamientos oncológicos en niños y adultos(24). En trauma, una patología habitualmente muy temida si no se dispone de sangre, la experiencia muestra que los resultados con Testigos de Jehová son similares a los de la población general.

Por lo expuesto, pensamos que:

1. Al rechazar la transfusión, los pacientes Testigos de Jehová no hacen otra cosa que ejercer su autonomía y libertad de decisión.

2. Es posible tratar a estos pacientes sin transfundirlos y los resultados tienen un respaldo científico sólido.

3. En la práctica, la vía legal estorba el trabajo médico y la relación médico-paciente.

\section{Programa de Atención en el Hospital Clínico de la Universidad de Chile}

Por esos motivos, desde 1998 se ha implementado en el Hospital Clínico de la Universidad de Chile un Programa de Atención (ver figura 1) para pacientes que no deseen transfundirse, que cuenta con adscripción voluntaria de profesionales de todas las especialidades médicas $(25,26)$. Este programa tiene como objetivos establecer estrategias, técnicas y procedimientos tendientes a optimizar la atención de estos pacientes, evitar someterlos a presiones innecesarias y contar con respaldo legal frente a acciones judiciales ejercidas. 
Figura 1: Diagrama de flujo del Programa de Medicina y Cirugía sin Transfusión de Sangre

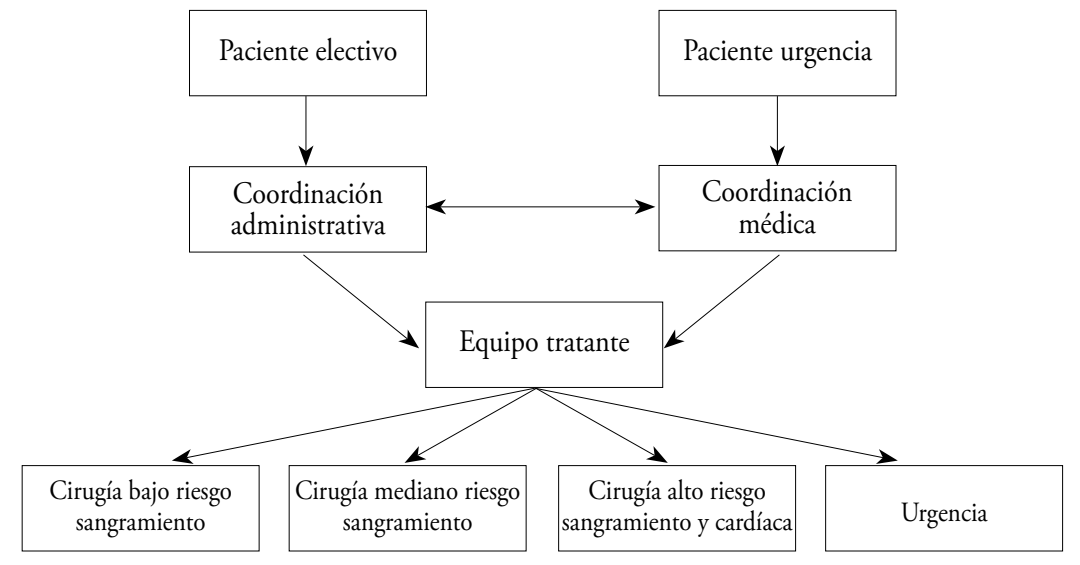

En Chile se han publicado resultados en cirugía cardía$\mathrm{ca}(27)$, cirugía traumatológica(28), traumatología en hemofílicos(29), cirugía ginecológica, urológica, trasplante renal y hepático, tratamiento oncológico(30), cirugía pediátrica(27) y de neonatología, aparte de los resultados globales de nuestro programa.

Acerca de las estrategias médico-quirúrgicas en la constitución del Programa, brevemente podemos señalar las siguientes:

- Tratamiento previo de la anemia y optimización de masa globular (estimulación eritropoyesis EPO; fierro)(31).

- Técnicas de mínima invasión (endoscopía, técnicas radiológicas, videoscopías, criocirugía, braquiterapia) (32,33).

- Hemodilución intraoperatoria.

- Técnicas quirúrgicas cuidadosas (control pedículos, posición, clampeo).

- Instrumental (electrobisturí, bisturí ultrasónico, láser radiofrecuencia).

- Fármacos (ácido tranexámico, aprotinina, desmopresina, EPO, Fierro IV, Factor VII recombinante(34-36).

- Agentes tópicos (adhesivos de colágeno, gelatinacelulosa, trombina tópica, gel plaquetario).

- Optimizar parámetros (temperatura y $\mathrm{Ph}$, volemia, hipotensión controlada)(37).

- Recuperación intraoperatoria de GR, restringir pérdidas (flebotomías), optimizar el transporte y minimizar consumo de $\mathrm{O} 2$ (38).

Respecto de los menores este programa tiene algunas limitaciones: se guía por el concepto mundial que reconoce el hecho de que un hijo de Testigos de Jehová pudiera no llegar a serlo cuando sea mayor, aunque sus padres lo sean. Esto no significa que a priori se desee contravenir la voluntad de los padres cuando los hijos son incompetentes. Se conversa con los padres y, finalmente, se aplican todos los conceptos de una buena medicina, lo que implica procurar que el paciente sea sometido al menor de los riesgos, tanto presentes como futuros.

Con relación a los menores competentes, una buena base la encontramos en el documento "Historia de la Ley 20.418", que regula tanto en adultos como en menores competentes la reproducción asistida. En dicho documento se establecen tres tramos en la adolescencia, “...que definen distintos grados de autonomía y requieren un manejo distinto por parte del profesional: adolescencia temprana, de los 10 a los 13 años de edad (...); adolescencia media, de los 14 a 16 años (...), y adolescencia tardía, entre los 17 y los 19 años, etapa que marca el fin de la adolescencia”. Esta es una buena guía para usar con menores competentes en menor o mayor grado, de modo que, cuando la ocasión lo amerite, también se incluya su parecer en la toma de decisiones para la recuperación de su salud.

En todos los casos, este Programa ha derivado en la mejor comprensión de los procesos fisiológicos de la anemia y ha propiciado el desarrollo de estrategias y técnicas que benefician a la población general y ayudan a los profesionales de la salud a efectuar una medicina moderna, basada en el respeto por la dignidad, autonomía y bienestar de los pacientes.

1 Disponible en: http://www.bcn.cl/histley/periodos?p=2010 Ultimo acceso en mayo 7, 2010. 


\section{Referencias}

1. Sagrera JE. La autonomía de los pacientes. JANO 2002; 62(1434): 10.

2. Consejo de Europa. Convenio para la Protección de los Derechos Humanos y la Dignidad del Ser Humano con respecto a las aplicaciones de la Biología y la Medicina. Aprobado por el Comité de Ministros el 19 de noviembre de 1996.

3. Ley de España № 41. Básica reguladora de la autonomía del paciente y de derechos y obligaciones en materia de información y documentación clínica.

4. International Society of Blood Transfusion (ISBT). Code of Ethics. Disponible en: http://www.isbt-web.org/documentation/default.asp

5. Retamales A. Lecciones que dejan los pacientes adultos que rechazan transfusiones de sangre a partir de la doctrina de nuestros tribunales. Ius Publicum 2003; 11: 75-102.

6. Rosengart TK, Helm RE, Klemperer J, Krieger KH, Waine Isom O. Uso combinado de aprotinina y eritropoyetina para la conservación de la sangre: Resultados con Testigos de Jehová. Ann Torca Surg 1994; 58: 1397-1403.

7. Van Son JAM, Hovaguimian H, Rao IM, He GW, Meiling GA, King DH et al. Strategies for repair of congenital heart defects in infants without the use of blood. The Society of Thoracic Surgeons 1995; 59: 384-388.

8. Cooley DA. Conservation of Blood During Cardiovascular Surgery. The American Journal of Surgery 1995; 170: 56-59.

9. Gozzetti G, Mazziotti A, Grazi GL, Jovine E, Gallucci A, Gruttadauria S, et al. Liver resection without blood transfusion. British Journal of Surgery 1995; 82: 1105-1110.

10. Jamieson GG, Corbel L, Campion JP, Launois B. Major liver resection without a blood transfusion: Is it a realistic objective? Surgery 1992; 112: 32-36.

11. Feagan BG, Wong CJ, Kirkley A, Johnston DWC, Smith FC, Whitsitt P, Wheeler SL, et al. Erythropoietin with iron supplementation to prevent allogeneic blood transfusion in total hip join arthroplasty. A randomized, controlled trial. Ann Intern Med 2000; 133: 845-854.

12. Meyers MO, Heinrich S, Kline R, Levine EA. Extended hemipelvectomy in a Jehovah's Witness with erythropoietin support. Am Surg 1998; 64: 1074-1076.

13. Nelson CL, Fontenot HJ. Ten Strategies to reduce Blood Loss in Orthopedic Surgery. The American Journal of Surgery 1995; 170: 64S-68S.

14. Thomas JM. La necesidad mundial de educación sobre el manejo sin sangre en obstetricia y ginecología. The Journal of the Society of Obstetricians and Gynaecologist of Canada 1994; 16: 1483-1487.

15. De Castro RM. Bloodless surgery: Establishment of a program for the special medical needs of the Jehovah's Witness community. The gynecologic surgery experience at a community hospital. Am J Obstet Gynecol 1999; 180: 14911498.

16. Monk TG, Goodnough LT, Brecher ME, Pulley DD, Colberg JW, Andriole GL, Catalona WJ. Acute normovolemic hemodilution can replace preoperative autologous blood donation as a Standard of care for autologous blood procurement in radical prostatectomy. Anesth Analg 1997; 85: 953-958.

17. Monk TG, Goodnough LT, Birkmeyer JD, Brecher ME, Catalona WJ. Acute normovolemic hemodilution is a costeffective alternative to preoperative autologous blood donation by patients undergoing radical retropublic prostatectomy. Transfusion 1995; 35: 559-565.

18. Corno AF, Laks H, Stevenson LW, Clark S, Drinkwater DC. Heart transplantation in a Jehovah's Witness. J Heart Transplantation 1986; 175-177.

19. Cacciarelli T, Keeffe EB, Moore DH, Burns W, Chuljian P, Busque S, et al. Primary liver transplantation without transfusion of red blood cells. Surgery 1996; 120: 698-705.

20. Ramos HC, Todo S, Kang Y, Felekouras E, Doyle HR, Starzl TE. Liver transplantation without the use of blood products. Arch Surg 1994; 129: 528-533.

21. Detry O, Honoré P, Delwaide J, Dondelinger RF, Meurisse M, Jacquet N. Liver Transplantation in a Jehovah's Witness. The Lancet 1999; 353: 1680.

22. Kaufman DB, Sutherland DER, Fryd DS, Ascher NL, Simmons RL, Najarian JS. A single-center experiencia of renal transplantation in thirteen Jehovah's Witnesses. Transplantation 1988; 45: 1045-1049.

23. Olugbenga AA, Custer JR, Bunchman TE, Sedman AB. Management of severa anemia without transfusión pediatric Jehovah's Witness patient. Critical Care Medicine 1994; 22: 524-528. 
24. Ballen KK, Ford PA, Witkus H, Emmons RVB Levy W, Doyle P, et al. Successful autologous bone marrow transplant without the use of blood product support. Bone Marrow Transplantation 2000; 26: 227-229.

25. Cardemil G, Braghetto I, Gonzáles M, Escobar C. Programa de atención médico-quirúrgica sin transfusiones sanguíneas. Revista Hospital Clinico Universidad de Chile 2000; 11: 212-221.

26. Cardemil G, Rodríguez F, Baeza F, Reyes D, Escobar C, Hepp M. Resultados del Programa de atención MédicoQuirúrgica sin uso de sangre ni hemoderivados del Hospital Clínico de la Universidad de Chile. Revista Chilena de Cirugía 2004; 56: 232-236.

27. Zamudio I. Manejo pre, intra y postoperatorio en la cirugía mayor sin transfusión sanguínea. Revista Chilena de Cirugía 2000; 52: 446-452.

28. Zamudio I, Solar P, Ortiz I, Arretz C, León L, Haecker S, et al. El uso de aprotinina en lactantes con D-TGA sometidos a la operación de Janete. Revista Chilena de Anestesia 1998; 27: 45-46.

29. Retamales A. Uso de la sangre y Testigos de Jehová. Capitulo Chileno de Medicina Transfusional 2002.

30. Rojas B, Meneses P. Testigo de Jehová portador de leucemia linfática aguda. Revista Chilena de Cancerología y Hematología 2000; 10: 151 .

31. Mesa redonda: Técnicas alternativas a las transfusiones perioperatorias. Revista Hospital Clínico Universidad de Chile 2000: $11 ; 329-337$.

32. Navarrete C. Manejo Endoscópico de la hemorragia digestiva. Revista Hospital Clínico Universidad de Chile 2000: 11; 318.

33. Cohn S. Alternativas técnicas e instrumentales del cirujano. Revista Hospital Clínico Universidad de Chile 2000: 11; 345-349.

34. Barón J.F. Alternativas farmacológicas en la cirugía sin sangre. Revista Hospital Clinico Universidad de Chile 2000: 11; 283-286.

35. Hedner U. Recombinant activated factor VII as a universal haemostatic agent. Blood Coagul Fibrinolysis 1998; 9(Supp1): S147-S152.

36. Vlot AJ. Treatment of a severely bleeding patient without preexisting coagulopathy with activated recombinant factor VII". Am J Surg 2000; 108(5): 421-423.

37. Krausz MM, Bashenko Y, Hirsh M. Crystalloid or colloid resuscitation of uncontrolled hemorrhagic shock after moderate splenic Injury. Shock 2000; 13: 230-235.

38. Shander A. Manejo del paciente en cuidados intensivos Revista Hospital Clínico Universidad de Chile 2000: 11; 363.

Recibido: 12 de marzo de 2010

Aceptado: 25 de marzo de 2010 